\title{
S100A16 up-regulates Oct4 and Nanog expression in cancer stem-like cells of Yumoto human cervical carcinoma cells
}

\author{
NARIAKI TOMIYAMA ${ }^{1,2}$, RYUJI IKEDA $^{1}$, YUKIHIKO NISHIZAWA ${ }^{1}$, \\ SHOGO MASUDA $^{1}$, YUSUKE TAJITSU ${ }^{2}$ and YASUO TAKEDA ${ }^{1}$ \\ ${ }^{1}$ Department of Clinical Pharmacy and Pharmacology, Graduate School of Medical and Dental Sciences, Kagoshima University, \\ Kagoshima 890-8520; ${ }^{2}$ Department of Pharmacy, Izumi General Medical Center, Izumi-shi, Kagoshima 899-0131, Japan
}

Received June 23, 2017; Accepted March 16, 2018

DOI: $10.3892 / 01.2018 .8568$

\begin{abstract}
Cancer stem-like cells (CSCs), which possess the ability to self-renewal and are multipotent, are regarded as the cause of tumor formation, recurrence, metastasis and drug resistance. It is necessary to understand the properties of CSCs in order to treat them effectively. It has been previously reported that S100 family proteins, which carry calcium-binding EF-hand motifs and are associated with tumorigenic processes, serve crucial roles in maintaining cancer stem-like properties. S100A16 is upregulated in various types of cancer, including bladder, lung and pancreatic. However, the roles of S100A16 in cancer cells, particularly CSCs, are not clear. The present study investigated the roles of S100A16 in CSCs using the sphere formation assay of Yumoto cells, which are a human cervical carcinoma cell line. The mRNA expression levels were evaluated by reverse transcription-polymerase chain reaction and the protein expression levels were detected by western blot analysis. Following the sphere formation of Yumoto cells, the mRNA and protein expression level of Oct4, Nanog and S100A16 were increased compared with the control cells. Following transfection with S100A16 small interfering RNA (siRNA), the mRNA and protein expression of Oct4 and Nanog were decreased and the spheroid size was significantly decreased in the sphere formation of Yumoto cells compared with control siRNA treated cells. There was no change in the $p 53$ mRNA expression level, whereas the $\mathrm{p} 53$ protein expression level, which was decreased by the sphere formation, was recovered by S100A16 knockdown. In addition, the protein expression levels of
\end{abstract}

Correspondence to: Professor Yasuo Takeda, Department of Clinical Pharmacy and Pharmacology, Graduate School of Medical and Dental Sciences, Kagoshima University, 8-35-1 Sakuragaoka, Kagoshima 890-8520, Japan

E-mail: takeda@m.kufm.kagoshima-u.ac.jp

Abbreviations: CSCs, cancer stem-like cells; EMT, epithelial mesenchymal transition; siRNA, small interfering RNA; RT-PCR, reverse transcription and polymerase chain reaction; ESCs, embryonic stem cells

Key words: cancer stem-like cells, S100A16, Oct4, Nanog, sphere formation assay
Oct4 and Nanog, which were increased in the sphere formation, were decreased by the proteasome inhibitor lactacystin. No differences were observed in the S100A16 protein expression between the presence or absence of lactacystin. These results suggest that S100A16 serves an important role in the CSCs of human cervical carcinoma and is a positive regulator of Oct4 and Nanog.

\section{Introduction}

The mortality of cancer is an important problem worldwide. Although many therapeutic strategies have been developed, they have not been sufficient for overcoming cancer. In particular, recurrence and treatment resistance make it difficult to treat cancer. Over the past decade, cancer stem-like cells (CSCs), which possess self-renewal and multipotency, are regarded as the cause of tumor formation, recurrence, metastasis, and drug resistance (1). Therefore, it is important to understand the properties of CSCs.

The several markers of CSCs have been reported, and Oct4 and Nanog play important roles in stem cells (2-4). Oct4, the homeodomain transcription factor of the POU family, is a key transcription factor for maintaining the self-renewal and pluripotency of embryonic stem cells (ESCs) (2). In addition, it has been reported that Oct4 plays a crucial role in the maintenance of dedifferentiation in CSCs (3). Similarly, Nanog, the homeobox domain transcription factor, is an essential regulator of ESCs (5). Both Oct4 and Nanog are essential to maintain stem cell properties (5), and Nanog appears to be one of the markers of CSCs (4).

CSCs are also known to possess a high efflux system, and express several adenosine triphosphate-binding cassette (ABC) transporters, such as P-glycoprotein (P-gp), breast cancer resistance protein (BCRP). Therefore, high expression of $\mathrm{ABC}$ transporters were common features of CSCs (6).

Recently, it has been reported that $\mathrm{S} 100$ proteins play crucial roles in maintaining cancer stem-like properties $(7,8)$. S100 is a family of calcium binding proteins of the EF-hand type that are involved in tumor development and progression, such as cell growth, migration invasion, tumor microenvironment, and stem properties $(7,8)$.

S100A16 is a novel protein of the S100 family that is up-regulated in various malignant tumors (9). It had been reported that S100A16 was associated with epithelial 
mesenchymal transition (EMT) and p53 tumor suppressor function $(10,11)$. The EMT plays crucial roles in the formation and differentiation of tissues. Recent studies suggest that EMT is involved in cancer metastasis, progression, and CSC properties $(7,12,13)$. These previous studies suggest that S100A16 plays an important role in CSCs. However, the role of S100A16 in cancer cells remains to be fully elucidated. In addition, the roles of S100A16 in CSCs also remain to be fully elucidated.

Therefore, we focused on S100A16 and investigated the roles of S100A16 in cancer cells by sphere formation assay.

\section{Materials and methods}

Cell culture. Yumoto, a human cervical squamous carcinoma cell line and has a wild-type p53 gene, was maintained in RPMI 1640 (Invitrogen; Thermo Fisher Scientific, Inc., Waltham, MA, USA) with $10 \%$ fetal bovine serum, $100 \mathrm{U} / \mathrm{ml}$ penicillin, $100 \mu \mathrm{g} / \mathrm{ml}$ streptomycin, and $0.25 \mu \mathrm{mg} / \mathrm{ml}$ amphotericin B at $37^{\circ} \mathrm{C}$ in $5 \% \mathrm{CO}_{2}$.

Sphere formation. Single-cell suspensions of Yumoto cells were seeded in serum-free Dulbecco's modified Eagle's medium/Nutrient Mix F-12 medium (1:1), containing GlutaMAX-I (DMEM/F12 + GlutaMAX; Invitrogen; Thermo Fisher Scientific, Inc.) medium with $20 \mathrm{ng} / \mathrm{ml}$ human recombinant epidermal growth factor (EGF) and $20 \mathrm{ng} / \mathrm{ml}$ human recombinant basic fibroblast growth factor (bFGF), cultured at a density of $1 \times 10^{6}$ cells $/ 5 \mathrm{ml} /$ dish in $60 \mathrm{~mm}$ ultra-low attachment dishes (Corning Incorporated, Corning, NY, USA) at $37^{\circ} \mathrm{C}$ in $5 \% \mathrm{CO}_{2}$ for 3 days.

RNA interference. S100A16 and negative control small interfering RNA (siRNA) duplexes were purchased from Qiagen $\mathrm{GmbH}$, (Hilden, Germany). Yumoto cells were transfected using the Lipofectamine RNAiMAX ${ }^{\mathrm{TM}}$ reagent (Invitrogen; Thermo Fisher Scientific, Inc.) according to the manufacturer's protocol. After the cells had incubated $24 \mathrm{~h}$ at $37^{\circ} \mathrm{C}$ in $5 \% \mathrm{CO}_{2}$, they were used in the following experiments.

Proteasome inhibition experiment. After the cells were cultured under the conditions of the sphere formation for 3 days, these cells were treated with $10 \mu \mathrm{M}$ lactacystin (Merck KGaA, Darmstadt, Germany) under the conditions of the sphere formation for $24 \mathrm{~h}$.

Isolation of RNA and reverse transcription and polymerase chain reaction $(R T-P C R)$ method. Total RNA was extracted using the TRIzol reagent according to the manufacturer's instructions (Thermo Fisher Scientific, Inc.). RT-PCR was performed with the SuperScript One-Step RT-PCR system (Thermo Fisher Scientific, Inc.) and gene-specific primers according to the manufacturer's instructions. Reaction mixtures contained total RNA (100 ng of each), $0.2 \mathrm{mM}$ dNTPs, $0.2 \mu \mathrm{M}$ of sense and antisense primers, an enzyme mixture including SuperScript II RT, Platinum Taq DNA polymerase, and 1x buffer with $1.2 \mathrm{mM} \mathrm{MgSO}_{4}$. The reaction was performed at $50^{\circ} \mathrm{C}$ for $20 \mathrm{~min}, 94^{\circ} \mathrm{C}$ for $2 \mathrm{~min}$, followed by $26-30$ cycles of $94^{\circ} \mathrm{C}$ for $15 \mathrm{sec}, 55^{\circ} \mathrm{C}$ for $30 \mathrm{sec}$, and $70^{\circ} \mathrm{C}$ for $30 \mathrm{sec}$. These sequences used the following primers: Oct4, 5'-(GTGGAG AGCAACTCCGATGGG)-3', and 5'-(CTCCACCCACTT
CTGCAGCAA)-3'; Nanog, 5'-(TCCAACATCCTGAACCTC AGC)-3', and 5'-(CTGGAACTGCATGCAGGACTG)-3'; P-gp, 5'-(TACAGCACGGAAGGCCTAATG)-3', and 5'-(TGT TCTCAGCAATGCTGCAGT)-3'; BCRP, 5'-(TCAGGAAGA CTTATGTTCCAC)-3', and 5'-(AGCTCTGTTCTGGATTCC AGT)-3'; multidrug resistance-associated protein 1 (MRP1), 5'-(GACACAGTGGACTCCATGATC)-3', and 5'-(CCACCA AGCCAGCACTGAGGC)-3'; p53, 5'-(CGCTGCTCAGAT AGCGATGGTCTGG)-3', and 5'-(GATTCTCTTCCTCTG TGCGCCGGTC)-3'; S100A16, 5'-(ACTGCTACACGGAGC TGGAGA)-3', and 5'-(GCAAGGGTCAGAGGAAGGTCT)-3'; GAPDH, 5'-(GCTCACTGGCATGGCCTTCCGTGTC)-3', and 5'-(CTCCTTGGAGGCCATGTGGGCCATG)-3'.

Cell extracts and western blotting. Cells were lysed with RIPA buffer (50 mM Tris-HCl, pH 8.0, $150 \mathrm{mM} \mathrm{NaCl}, 1 \%$ Nonidet P-40 (NP-40), 0.1\% SDS, 0.5\% sodium deoxycholate) containing protease inhibitor (complete EDTA-free; Roche Diagnostics, Indianapolis, IN, USA) and centrifuged at $12,000 \mathrm{rpm}$ for $10 \mathrm{~min}$ at $4^{\circ} \mathrm{C}$. The total cell extracts (50 $\mu \mathrm{g}$ each) were separated by $15 \%$ SDS-polyacrylamide gel electrophoresis and transferred to polyvinylidene difluoride membrane (Bio-Rad Laboratories, Inc., Hercules, CA, USA) for S100A16, Oct4, Nanog, p53, and GAPDH detection. The membranes were washed in Tris-buffered saline with Tween 20 (TBS-T, composed of $10 \mathrm{mM}$ Tris- $\mathrm{HCl}, \mathrm{pH} \mathrm{8,} 150 \mathrm{mM} \mathrm{NaCl}$, and $0.05 \%$ Tween 20), blocked for $1 \mathrm{~h}$ at room temperature with $5 \%$ nonfat milk in TBS-T, and probed overnight at $4^{\circ} \mathrm{C}$ with anti-S100A16 (Sigma-Aldrich; Merck KGaA, Darmstadt, Germany), anti-Oct4 (Bioworld Technology, Inc., St. Louis Park, MN, USA), anti-Nanog (Abcam, Cambridge, UK), anti-p53 (Santa Cruz Biotechnology, Inc., Dallas, TX, USA), and anti-GAPDH (Cell Signaling Technology, Inc., Danvers, MA, USA) antibodies. After incubation for $1 \mathrm{~h}$ at room temperature with horseradish peroxide-conjugated secondary antibody, immunoreactive proteins were detected by the ECL detection system (GE Healthcare Life Sciences, Little Chalfont, UK).

Measurement of spheroid size in sphere formation assay. The spheroid size at day 3 in the sphere formation assay was measured by microscopy and calibration slide. The spheroid size was defined as the average spheroid diameter of 100 spheroids.

Statistical analysis. Differences between groups were tested by Student's t-test. Data are presented as means \pm SD. Differences were considered significant at $\mathrm{P}<0.01$.

\section{Results}

Expression levels of Oct4, Nanog, ABC transporter, and S100A16 in sphere formation of Yumoto cells. To confirm the properties of the sphere formation of Yumoto cells, the expression levels of Oct4, Nanog, ABC transporter, and S100A16 were examined. As shown in Figs. 1 and 2, the expression levels of Oct4, Nanog, BCRP, MRP1, and S100A16 increased in the sphere formation of Yumoto cells compared with the monolayer cultured cells. These data show that the expression of S100A16 up-regulates in the sphere formation of Yumoto cells. 
A

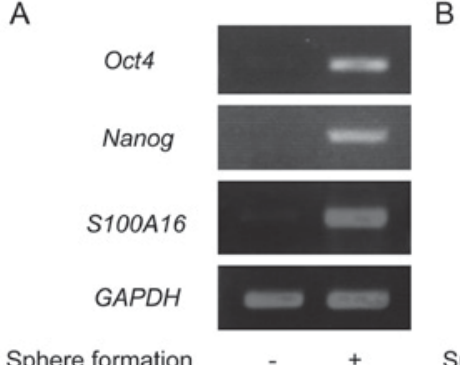

B

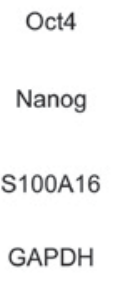

Sphere formation
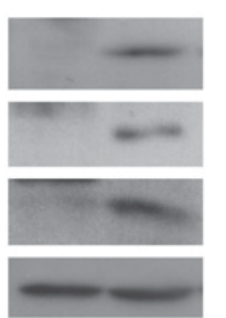

$+$

Figure 1. The expression levels of Oct4, Nanog, and S100A16 in the sphere formation of Yumoto cells. The cells were cultured under the non-adherence and serum-free culture conditions of the sphere formation assay for 3 days. (A) Total RNA was extracted, and the expressions of Oct4, Nanog, and S100A16 were evaluated by reverse transcription-polymerase chain reaction. (B) The cell lysates were prepared from these cells, and the expressions of Oct4, Nanog, and S100A16 were detected by immunoblotting using antibodies against Oct4, Nanog, and S100A16. Oct4, octamer-binding transcription factor 4; Nanog, homeobox protein NANOG.

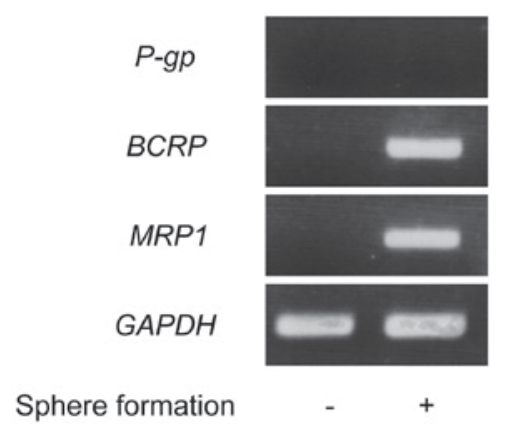

Figure 2. The expression levels of $P$ - $g p, B C R P$, and $M R P 1$ in the sphere formation of Yumoto cells. The cells were cultured under the non-adherence and serum-free culture conditions of the sphere formation assay for 3 days. Total RNA was extracted, and the expressions of $P-g p, B C R P$, and $M R P 1$ were evaluated by reverse transcription-polymerase chain reaction. $M R P 1$, multidrug resistance-associated protein 1; $P$-gp, P-glycoprotein; $B C R P$, breast cancer resistance protein.

Effect of S100A16-targeting siRNA on spheroid forming in sphere formation of Yumoto cells. Only CSCs formed spheroids and survived in the non-adherence and serum-free culture conditions of the sphere formation assay. To examine whether S100A16 regulates the spheroid-like body formation of Yumoto cells, the S100A16 or control siRNA treated cells were cultured under the non-adherence and serum-free culture conditions of the sphere formation assay for 3 days. Fig. 3 shows the effect of S100A16 siRNA on spheroid forming in the sphere formation of Yumoto cells over the 3 days. The spheroid size was significantly decreased in S100A16 siRNA treated cells compared with control siRNA treated cells. These data indicate that S100A16 is involved in spheroid forming in the sphere formation of Yumoto cells.

Effect of S100A16-targeting siRNA on expression of Oct4, Nanog, and p53 in sphere formation of Yumoto cells. Fig. 4 shows the effect of S100A16-targeting siRNA on the expression levels of Oct4, Nanog, and p53 in the sphere formation of Yumoto cells evaluated by RT-PCR and immunoblotting. In the sphere formation of Yumoto cells, the expression levels of Oct4 and Nanog were decreased in S100A16 siRNA treated

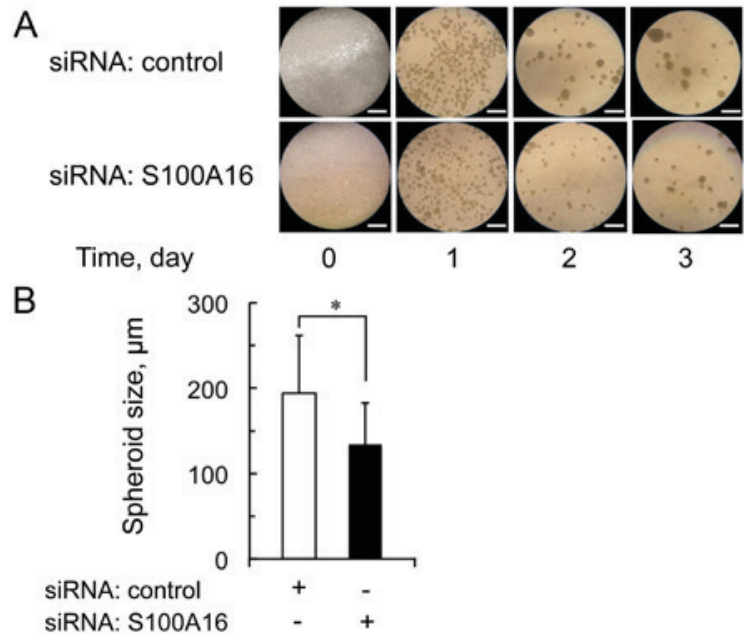

Figure 3. Effect of S100A16-targeting siRNA on spheroid forming in the sphere formation of Yumoto cells. These cells were transfected with S100A16-targeting or control siRNA. After these cells were incubated for $24 \mathrm{~h}$, the cells were cultured under the conditions of the sphere formation assay for 3 days. (A) The microphotographs of these cells' morphological changes in sphere formation are shown at various time points $(0,1,2$, and 3 days). Scale bar, $500 \mu \mathrm{m}$. (B) The average spheroid size at day 3 was measured by microscopy and calibration slide. The data shown represent the mean \pm standard deviation. ${ }^{*} \mathrm{P}<0.01$. siRNA, small interfering RNA.
A

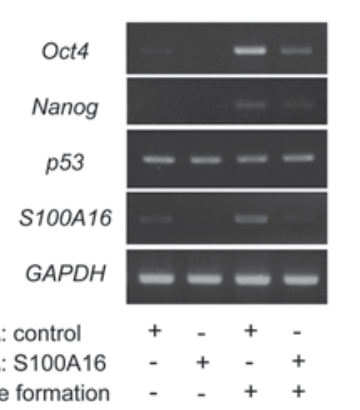

B

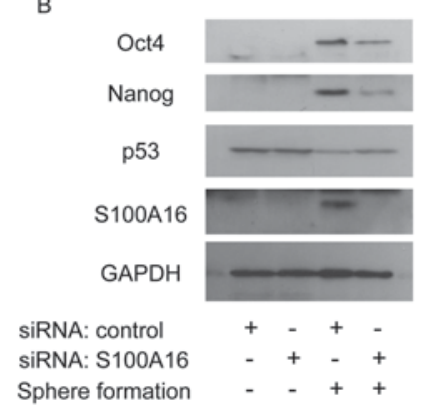

Figure 4. Effect of S100A16-targeting siRNA on the expression of Oct4, Nanog, and p53 in the sphere formation of Yumoto cells. The cells were transfected with S100A16-targeting or control siRNA. After these cells were incubated for $24 \mathrm{~h}$, these cells were cultured under the conditions of the sphere formation assay for 3 days. (A) Total RNA was extracted, and the expression levels of Oct4, Nanog, p53, and S100A16 were evaluated by reverse transcription-polymerase chain reaction. (B) The cell lysates were prepared from these cells, and the expression levels of Oct4 Nanog, p53, and S100A16 were detected by immunoblotting using antibodies against Oct 4 , Nanog, p53, and S100A16. Oct4, octamer-binding transcription factor 4; Nanog, homeobox protein NANOG; siRNA, short interfering RNA.

cells compared with control siRNA treated cells. These data suggest that S100A16 up-regulates Oct4 and Nanog expression in the CSCs of Yumoto cells.

It has been reported that the expressions of Oct 4 and Nanog are regulated by p53 and that S100A16 interacts with p53 $(11,14)$. In order to confirm whether p53 was involved in the up-regulation of Oct4 and Nanog by S100A16, the expression level of p53 was examined in the sphere formation of Yumoto cells after transfection with S100A16 siRNA. As shown in Fig. 4, there was no change in the mRNA expression level of $p 53$, whereas the protein expression level of $\mathrm{p} 53$, which was decreased in the sphere formation, was recovered by S100A16 knockdown. Therefore, the up-regulation of Oct 4 and Nanog 


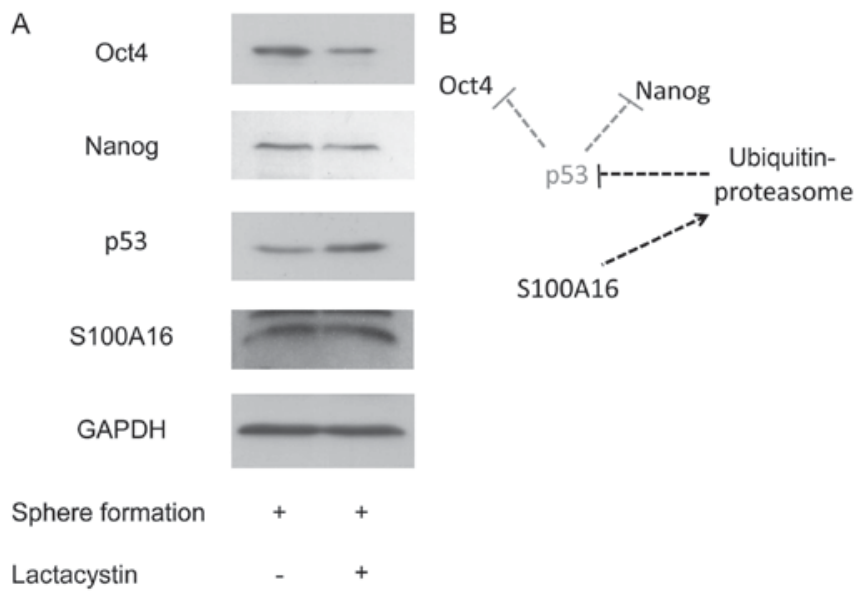

Figure 5. (A) Effect of proteasome inhibition on the expressions of Oct4, Nanog, p53, and S100A16 in the sphere formation of Yumoto cells. After the cells were cultured under the conditions of the sphere formation assay for 3 days, these cells were treated with proteasome inhibitor lactacystin $(10 \mu \mathrm{M})$ under the conditions of the sphere formation assay for $24 \mathrm{~h}$. The cell lysates were prepared from these cells, and the expression levels of Oct4 Nanog, p53, and S100A16 were detected by immunoblotting using antibodies against Oct4, Nanog, p53, and S100A16. (B) Potential schematic diagram of the effect of S100A16 on the expression of Oct4 and Nanog expression in CSCs of Yumoto human cervical carcinoma cells. Oct4, octamer-binding transcription factor 4; Nanog, homeobox protein NANOG.

by S100A16 seems to be mediated by suppressing p53 in the sphere formation of Yumoto cells.

Effect of proteasome inhibition on expression of Oct4, Nanog, p53, and S100A16 in sphere formation of Yumoto cells. It has been reported that some S100 family members interact with p53 and promote proteasome-dependent degradation of p53 $(7,8,15,16)$. In order to confirm whether S100A16 was involved in p53 proteasome degradation, proteasome inhibition by lactacystin was examined in the sphere formation of Yumoto cells. As shown in Fig. 5A, the protein expression level of p53 was increased by lactacystin. The protein expression levels of Oct4 and Nanog, which were increased in the sphere formation, were decreased by lactacystin, whereas no difference was observed in the S100A16 protein levels between the presence or absence of lactacystin. The spheroid size was not affected by the addition of lactacystin. The results suggested that S100A16 may up-regulate Oct4 and Nanog expression to promote p53 degradation in the CSCs of Yumoto cells (Fig. 5B).

\section{Discussion}

We have demonstrated in this study that the S100A16 gene positively regulated the expressions of Oct4 and Nanog in the sphere formation of Yumoto cells. This is the first report that S100A16 is involved in the expression levels of Oct4 and Nanog in CSCs.

In the present study, we used the sphere formation assay to evaluate CSCs. The sphere formation assay is based on the mechanism by which only CSCs survive, by forming spheroids, under non-adherence and serum-free culture conditions (17). The sphere formation assay is particularity useful to enrich the potential CSC subpopulations. Furthermore, numerous studies have demonstrated purported CSCs by the sphere formation assay (17-19).

It has been reported that Oct 4 expression maintained cancer stem-like properties, and treatment with Oct 4 siRNA resulted in decreased spheroid size in the sphere formation $(3,20)$. The CSCs survived by forming spheroids in the culture conditions of the sphere formation assay, whereas non CSCs did not survive and did not form spheroids. Therefore, the suppression of the Oct4 expression after treatment with S100A16 siRNA affects the cancer stem cell-like properties and reduces the number of viable cells in the culture conditions of the sphere formation of Yumoto cells.

The tumor suppressor protein, p53, is an important factor for the cell cycle and apoptosis, and its dysfunction is associated with increased tumor development $(21,22)$. It has been reported that Oct 4 and Nanog were negatively regulated by p53 (14), and p53 was negatively regulated by the interaction of S100A16 with p53 (11). It has been reported that S100 protein family members interact with p53 and affect p53 function (7-9,15). In particular, S100A4 interacts with p53 and promotes $\mathrm{p} 53$ proteasome degradation (16). In the present study, the protein expression levels of Oct4 and Nanog, which were increased in the sphere formation, were decreased by lactacystin, whereas no difference was observed in the S100A16 protein levels between the presence or absence of lactacystin. Although p53 is the frequently mutated tumor suppressor gene in human cancer $(21,22)$, the Yumoto cell line has a wild-type $p 53$ gene (23). Hence, the results suggested that S100A16 may up-regulate Oct4 and Nanog to promote p53 degradation in the CSCs of Yumoto cells (Fig. 5B). It has been reported that a number of $\mathrm{S} 100$ proteins affect p53 function through several mechanisms, such as inhibition of p53 phosphorylation, modulation of the p53 oligomerization state, and p53 degradation $(2,3)$. Further studies by other methods are needed to elucidate the mechanism for the regulation of $\mathrm{p} 53$ function by S100A16.

Regulation of Oct4 and Nanog by the ubiquitin-proteasome system and Oct4 and Nanog up-regulation by proteasome inhibition in human embryonic stem cells has been reported $(24,25)$. These previous reports were opposite to our result that Oct4 and Nanog were down-regulated by the proteasome inhibitor. However, it has been reported that proteasome activity is down-regulated in CSCs (26). Thus, the regulation of Oct4 and Nanog expression may be more affected by p53 than the ubiquitin-proteasome system in the CSCs of Yumoto cells.

In the present study, Yumoto human cervical carcinoma cells are used for experiments. There are no reports on the expression of S100A16 in cervical carcinoma. It has been reported that a high S100A16 expression level is correlated to poor prognosis in breast cancer and lung adenocarcinoma $(27,28)$, whereas a low S100A16 expression level is correlated with reduced survival and poor tumor differentiation in oral squamous cell carcinoma (29). Further studies are needed to clarify the function of S100A16 and its clinical significance in the various cancer types.

In conclusion, our data indicates that S100A16 is a positive regulator of Oct4 and Nanog in the CSCs of Yumoto human cervical carcinoma cells and that S100A16 may play important roles in the CSCs of Yumoto human cervical carcinoma cells. 


\section{Acknowledgements}

The authors would like to thank Chizuko Nakamura for their support and advice at Gyokusui-Kai Hospital.

\section{Funding}

The present study was supported by JSHP KAKENHI (grant no. 26460201).

\section{Availability of data and materials}

All data generated or analyzed during this study are included in this published article.

\section{Authors' contributions}

NT designed the study and wrote the initial draft of the manuscript. RI contributed to the analysis and interpretation of the data, and assisted in the preparation of the manuscript. YN, SM, YuT and YaT contributed to data collection and interpretation and critically reviewed the manuscript. All authors read and approved the final manuscript.

\section{Ethics approval and consent to participate}

Not applicable.

\section{Consent for publication}

Not applicable.

\section{Competing interests}

The authors declare that they have no competing interests.

\section{References}

1. Jordan CT, Guzman ML and Noble M: Cancer stem cells. N Engl J Med 355: 1253-1261, 2006.

2. Rosner MH, Vigano MA, Ozato K, Timmons PM, Poirier F, Rigby PW and Staudt LM: A POU-domain transcription factor in early stem cells and germ cells of the mammalian embryo. Nature 345: 686-692, 1990.

3. Kumar SM, Liu S, Lu H, Zhang H, Zhang PJ, Gimotty PA, Guerra M, Guo W and Xu X: Acquired cancer stem cell phenotypes through Oct4-mediated dedifferentiation. Oncogene 31: 4898-4911, 2012.

4. Jeter CR, Yang T, Wang J, Chao HP and Tang DG: Concise review: NANOG in cancer stem cells and tumor development: An update and outstanding questions. Stem Cells 33: 2381-2390, 2015.

5. Chambers I, Colby D, Robertson M, Nichols J, Lee S, Tweedie S and Smith A: Functional expression cloning of Nanog, a pluripotency sustaining factor in embryonic stem cells. Cell 113: 643-655, 2003.

6. Savage P: Chemotherapy curable malignancies and cancer stem cells: A biological review and hypothesis. BMC Cancer 16: 906, 2016.

7. Chen $\mathrm{H}, \mathrm{Xu} \mathrm{C}$, Jin Q and Liu Z: S100 protein family in human cancer. Am J Cancer Res 4: 89-115. 2014.

8. Bresnick AR, Weber DJ and Zimmer DB: S100 proteins in cancer. Nat Rev Cancer 15: 96-109, 2015.

9. Marenholz I and Heizmann CW: S100A16, a ubiquitously expressed EF-hand protein which is up-regulated in tumors. Biochem Biophys Res Commun 313: 237-244, 2004.
10. Zhou W, Pan H, Xia T, Xue J, Cheng L, Fan P, Zhang Y, Zhu W, Xue Y, Liu X, et al: Up-regulation of S100A16 expression promotes epithelial-mesenchymal transition via Notch1 pathway in breast cancer. J Biomed Sci 21: 97, 2014.

11. Liu Y, Zhang R, Xin J, Sun Y, Li J, Wei D and Zhao AZ: Identification of S100A16 as a novel adipogenesis promoting factor in 3T3-L1 cells. Endocrinology 152: 903-911, 2011.

12. Chiou SH, Wang ML, Chou YT, Chen CJ, Hong CF, Hsieh WJ, Chang HT, Chen YS, Lin TW, Hsu HS and Wu CW: Coexpression of Oct4 and Nanog enhances malignancy in lung adenocarcinoma by inducing cancer stem cell-like properties and epithelial-mesenchymal transdifferentiation. Cancer Res 70: 10433-10444, 2010.

13. Thiery JP, Acloque H, Huang RY and Nieto MA: Epithelial-mesenchymal transitions in development and disease. Cell 139: 871-890, 2009.

14. Abdelalim EM and Tooyama I: Knockdown of p53 suppresses Nanog expression in embryonic stem cells. Biochem Biophys Res Commun 443: 652-657, 2014.

15. Lin J, Yang Q, Wilder PT, Carrier F and Weber DJ: The calcium-binding protein S100B down-regulates p53 and apoptosis in malignant melanoma. J Biol Chem 285: 27487-27498, 2010.

16. Orre LM, Panizza E, Kaminskyy VO, Vernet E, Gräslund T, Zhivotovsky B and Lehtiö J: S100A4 interacts with p53 in the nucleus and promotes p53 degradation. Oncogene 32: 5531-5540, 2013.

17. Pastrana E, Silva-Vargas V and Doetsch F: Eyes wide open: A critical review of sphere-formation as an assay for stem cells. Cell Stem Cell 8: 486-498, 2011.

18. Wei B, Han XY, Qi CL, Zhang S, Zheng ZH, Huang Y, Chen TF and Wei HB: Coaction of spheroid-derived stem-like cells and endothelial progenitor cells promotes development of colon cancer. PLoS One 7: e39069, 2012.

19. Fujii H, Honoki K, Tsujiuchi T, Kido A, Yoshitani K and Takakura Y: Sphere-forming stem-like cell populations with drug resistance in human sarcoma cell lines. Int J Oncol 34: 1381-1386, 2009.

20. Chen YC,HsuHS,Chen YW,TsaiTH,How CK, Wang CY,HungSC, Chang YL, Tsai ML, Lee YY, et al: Oct-4 expression maintained cancer stem-like properties in lung cancer-derived CD133-positive cells. PLoS One 3: e2637, 2008.

21. Hollstein M, Sidransky D, Vogelstein B and Harris CC: p53 mutations in human cancers. Science 253: 49-53, 1991.

22. Levine AJ, Momand J and Finlay CA: The p53 tumour suppressor gene. Nature 351: 453-456, 1991.

23. Naniwa J, Kigawa J, Akeshima R, Kanamori Y, Itamochi H, Oishi T, Iba T and Terakawa N: Leptomycin B enhances CDDP-sensitivity via nuclear accumulation of $\mathrm{p} 53$ protein in HPV-positive cells. Cancer Sci 94: 1099-1103, 2003.

24. Xu HM, Liao B, Zhang QJ, Wang BB, Li H, Zhong XM, Sheng HZ, Zhao YX, Zhao YM and Jin Y: Wwp2, an E3 ubiquitin ligase that targets transcription factor Oct-4 for ubiquitination. J Biol Chem 279: 23495-23503, 2004.

25. Floyd ZE, Staszkiewicz J, Power RA, Kilroy G, Kirk-Ballard H, Barnes CW, Strickler KL, Rim JS, Harkins LL, Gao R, et al: Prolonged proteasome inhibition cyclically upregulates Oct $3 / 4$ and Nanog gene expression, but reduces induced pluripotent stem cell colony formation. Cell Reprogram 17: 95-105, 2015.

26. Vlashi E, Kim K, Lagadec C, Donna LD, McDonald JT, Eghbali M, Sayre JW, Stefani E, McBride W and Pajonk F: In vivo imaging, tracking, and targeting of cancer stem cells. J Natl Cancer Inst 101: 350-359, 2009.

27. Tanaka M, Ichikawa-Tomikaw N, Shishito N, Nishiura K, Miura T, Hozumi A, Chiba H, Yoshida S, Ohtake T and Sugino T: Co-expression of S100A14 and S100A16 correlates with a poor prognosis in human breast cancer and promotes cancer cell invasion. BMC Cancer 13: 53, 2015.

28. Saito K, Kobayashi M, Nagashio R, Ryuge S, Katono K, Nakashima H, Tsuchiya B, Jiang SX, Saegusa M, Satoh Y, et al: S100A16 is a prognostic marker for lung adenocarcinomas. Asian Pac J Cancer Prev 16: 7039-7044, 2015.

29. Sapkota D, Bruland O, Parajuli H, Osman TA, Teh MT, Johannessen AC and Costea DE: S100A16 promotes differentiation and contributes to a less aggressive tumor phenotype in oral squamous cell carcinoma. BMC Cancer 15: $631,2015$. 